\section{ORIGINAL RESEARCH}

J. Wang

H. You

J.-F. Liu

D.-F. Ni

Z.-X. Zhang

J. Guan

\title{
Association of Olfactory Bulb Volume and Olfactory Sulcus Depth with Olfactory Function in Patients with Parkinson Disease
}

BACKGROUND AND PURPOSE: Olfactory dysfunction is commonly associated with IPD. We here report the association of $\mathrm{OB}$ volume and $\mathrm{OS}$ depth with olfactory function in patients with PD.

MATERIALS AND METHODS: Morphometric analyses by using MR imaging and the Japanese T\&T olfactometer threshold test were used to evaluate olfactory structure and function in 29 patients with PD and 29 age- and sex-matched healthy controls.

RESULTS: The olfactory recognition thresholds were significantly higher in patients with PD than in healthy controls $(3.82 \pm 1.25$ versus $0.45 \pm 0.65, P<.001)$. Olfactory atrophy with reductions in the volume of the OB $\left(37.30 \pm 10.23 \mathrm{~mm}^{3}\right.$ versus $\left.44.87 \pm 11.84 \mathrm{~mm}^{3}, P<.05\right)$ and in the depth of OS $(8.90 \pm 1.42 \mathrm{~mm}$ versus $9.67 \pm 1.24 \mathrm{~mm}, P<.05)$ was observed in patients with PD but not in controls. Positive correlations between olfactory performance and $\mathrm{OB}$ volumes were observed in both patients with PD $(r=-0.45, P<.0001)$ and in controls $(r=-0.42, P<.0001)$. In contrast, there was no significant correlation between the depth of OS and olfactory function in either cohort.

CONCLUSIONS: The results provide evidence that early olfactory dysfunction in patients with PD may be a primary consequence of damage to the OB. Neuroimaging of olfactory structures together with the assessment of olfactory function may be used to identify patients with PD.

ABBREVIATIONS: DWI = diffusion-weighted imaging; IPD = idiopathic Parkinson disease; $\mathrm{OB}=$ olfactory bulb; OS = olfactory sulcus; PD = Parkinson disease; $S E M=$ standard error of the mean; $\mathrm{SNpc}=$ substantia nigra pars compacta; $\mathrm{T} \& \mathrm{~T}=$ Toyota and Takagi

$\mathbf{0}$ lfactory deficits are common in patients with IPD, occurring at about the same frequency as resting tremor. ${ }^{1-3} \mathrm{Be}-$ tween $70 \%$ and $90 \%$ of patients with PD have olfactory deficits independent of disease severity and duration. ${ }^{4,5}$ Olfactory dysfunction is thus the second most common feature of this disorder, following rigidity and akinesia. ${ }^{6}$ The high prevalence of olfactory dysfunction in patients with IPD suggests that IPD may actually be an olfactory disease. ${ }^{5,6}$ Recent neuropathologic advances suggest that the olfactory system is among the earliest brain regions involved in $\mathrm{PD}^{7}$ and olfactory deficits are associated with the presence of incidental Lewy bodies in the brains of decedents without parkinsonism or dementia during life. ${ }^{8}$ Results from postmortem studies revealed Lewy bodies in the $\mathrm{OB}^{9}$ but also in other brain regions related to olfaction, such as the anterior olfactory nucleus, ${ }^{10}$ the piriform cortex, ${ }^{11}$ the amygdaloid complex, ${ }^{12}$ the entorhinal cortex, and the hippocampal formation. ${ }^{12}$ A recent study demonstrated that olfactory dysfunction in PD is related to atrophy in olfactoryeloquent regions of the limbic and paralimbic cortices. ${ }^{13}$ These olfactory deficits have been linked to structural and/or functional changes at the level of the OB ${ }^{9,12,14,15}$ and OS. ${ }^{16}$

There is evidence from numerous studies that MR imaging can be used to reliably evaluate the volume of the $\mathrm{OB}$ and the

Received May 11, 2010; accepted after revision September 7

From the Department of Otolaryngology, Peking Union Medical College Hospital, Beijing, China

Jian Wang and Hui You contributed equally to this work.

Please address correspondence to Dao-Feng Ni, Prof. MD, Department of Otolaryngology, Peking Union Medical College Hospital, Beijing 100730, China; e-mail: nidf@csc. pumch.ac.cn

DOI 10.3174/ajnr.A2350 depth of OS. ${ }^{15-18}$ It has been suggested that analysis of OB volume might be helpful in differential and early diagnosis of PD. ${ }^{15,18}$ However, recent studies did not show significant differences in $\mathrm{OB}$ volume between patients with $\mathrm{PD}$ and healthy controls. ${ }^{15,18}$ These results are surprising in light of the significant decrease in dopaminergic neurons in the anterior olfactory nucleus, which is part of the OB. ${ }^{19}$ Moreover, the depths of the bilateral OS measured from the coronal view in MR imaging were not different between the patients with PD and control groups. ${ }^{16}$ Therefore, it was unclear whether OB structural changes are associated with olfactory dysfunction in PD.

This study, therefore, set out to assess the OB volumes and OS depths and their correlations with olfactory function in patients with PD and in healthy controls. Olfactory function was correlated with reductions in the volume of $\mathrm{OB}$ and the depth of OS in patients with PD. Therefore, neuroimaging of the olfactory structure together with the assessment of olfactory function may be relevant to PD diagnosis.

\section{Materials and Methods}

\section{Subjects}

A total of 30 patients with PD (15 men and 15 women; mean age, 61.7 years; range, 43-78 years) and 30 age- and sex-matched healthy controls (15 men and 15 women; mean age, 62.6 years, range, 42-81 years) were initially included in the study. The volume of the OB in the 19th patient with PD was more than the value of mean $\pm 3 \mathrm{SD}$, thus this sample and the matched control were excluded. Therefore, there were only 29 patients with PD and 29 healthy controls included for statistical analysis. All participants were recruited through the Department of Neurology at the University of Peking Union Medical College Hospital. Diagnoses were made according to the diagnostic 

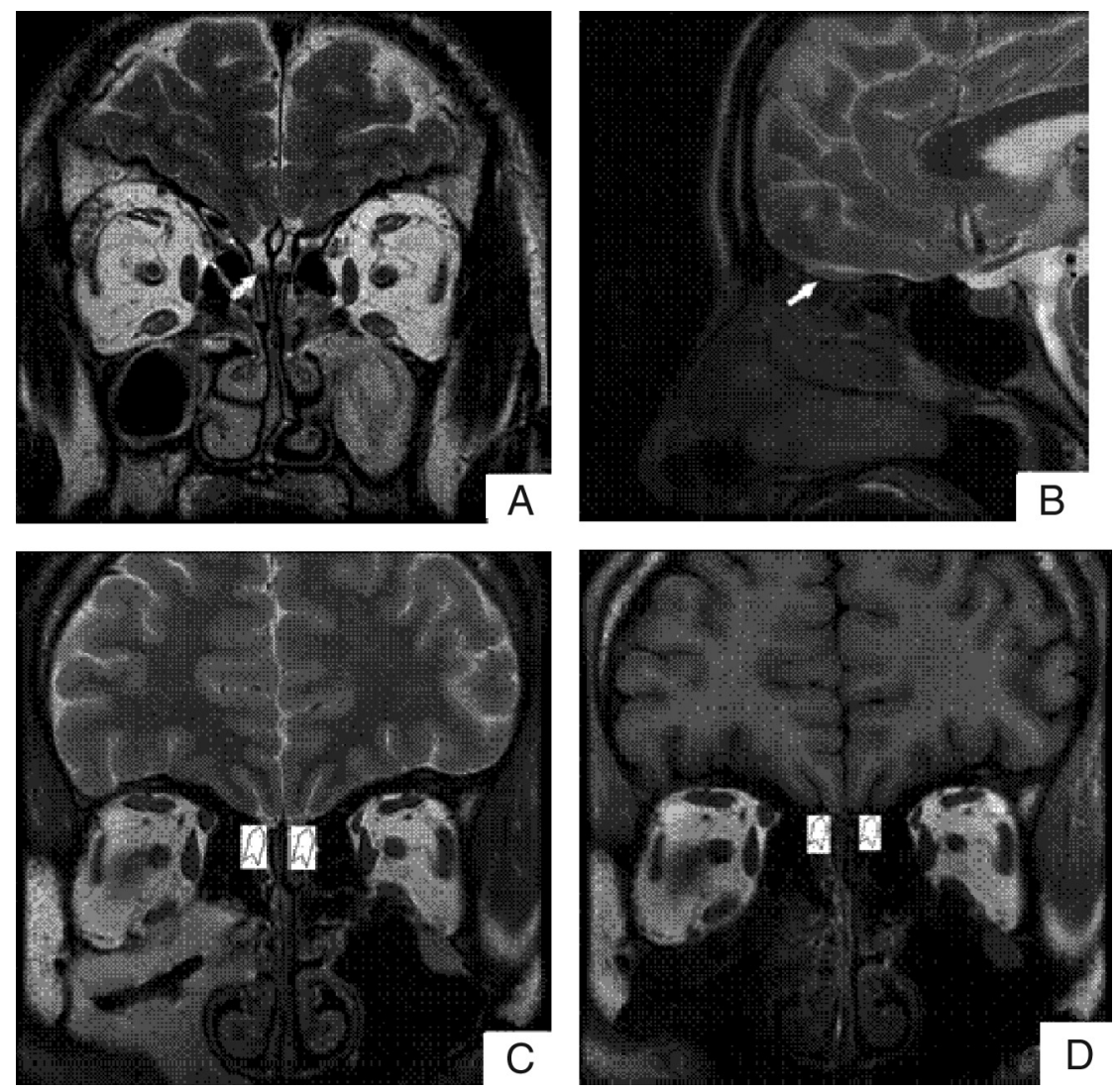

Fig 1. Visualization of the $\mathrm{OB}$ and the $\mathrm{OS}$ on representative MR images. Coronal $(A)$ and sagittal $(B)$ views of the brain show the OB (white arrows). Coronal T2-weighted $(C)$ and T1-weighted (D) images show the OS (white arrowhead).

criteria for PD. ${ }^{20}$ All of these patients were regularly evaluated in the outpatient department or during in-hospital stays. None of the healthy control subjects had a history of or signs of major neurologic or rhinologic disorders. Written informed consent was obtained from all participants. The study was approved by the Ethics Committee of the University of Peking Union Medical College Hospital and was performed according to the International Ethical Guidelines for Biomedical Research Involving Human Subjects.

\section{Olfactory Threshold Test}

The Japanese T\&T olfactometer test kit (Takasago Industry, Tokyo, Japan) includes 5 odorants, including $\beta$-phenylethyl alcohol, cyclotene, isovaleric acid, $\gamma$-undecalactone, and scatole. Each odorant is evaluated at 8 levels ( -2 to 5 ) with the exception of cyclotene, which is evaluated at 7 ( -2 to 4$)$. This test kit was used to determine detection and recognition thresholds for each of the odorants to evaluate bilateral olfactory ability and the degree of smell impairment as previously described. ${ }^{21}$ The detection threshold was defined as the lowest concentration detectable by the subject, whereas the recognition threshold was defined as the lowest concentration at which the odor can be identified. The testing process is a stepwise procedure, in which odorant concentrations are increased or decreased on the basis of the subject's responses. The detection and recognition thresholds for the 5 odorants were averaged for each subject, and the values were used to evaluate the olfactory acuities.

\section{MR Imaging}

MR imaging was performed by using a 3T system (Signa VH/i Excite II 3T; GE Healthcare, Milwaukee, Wisconsin) with a standard quadrature head coil to detect the $\mathrm{OB}$ anatomy as described previously. ${ }^{22,23}$ Coronal T1-weighted or T2-weighted spin-echo sequences were performed with the following parameters: 600/20 ms (TR/TE); section thickness, $3 \mathrm{~mm}$; matrix, $256 \times 256$; FOV, $120 \times 120 \mathrm{~mm}$; and the in-plane pixel resolution, $0.47 \times 0.47 \mathrm{~mm}$. Sections were angulated perpendicular to the anterior base of skull or cribriform plate. The observed region started at the frontal paranasal sinus and ended dorsally behind the crura cerebri. Entire brains were imaged with a T1-weighted 3D magnetization-prepared rapid acquisition of gradient echo imaging sequence with the following parameters: 9.7/4 ms (TR/TE); flip angle, $12^{\circ}$; and isotropic voxels, $1.0 \times 1.0 \times 1.0 \mathrm{~mm}$. Multiplanar reformation was conducted in 3 orthogonal planes. Saggital T2-weighted sequences were also performed.

\section{Imaging Data Analyses}

Acquired datasets were transferred to a workstation (GE Advantage Windows 4.2). Volume and depth measurements were performed by an experienced radiologist by manual segmentation of the coronal sections through the OBs. The OB and OS were manually measured by using routine coronal T1-weighted MR imaging sections (Fig 1). The boundaries of the $\mathrm{OB}$ were determined by using the surrounding $\mathrm{CSF}$ and the anterior cribriform plate as markers. The bilateral volume of the OB and the depth of the OS were measured in all study subjects. Because there may be differences between left- and rightsided $\mathrm{OB}$ volumes and OS depth, ${ }^{22-24}$ we used the maximum volume of the $\mathrm{OB}$ and depth of the OS in each individual for the analysis. The $\mathrm{OB}$ volume was expressed in cubic millimeters by calculating the inplane area and the thickness of the OB. All the measurements were done by an individual who was blinded to diagnoses. 


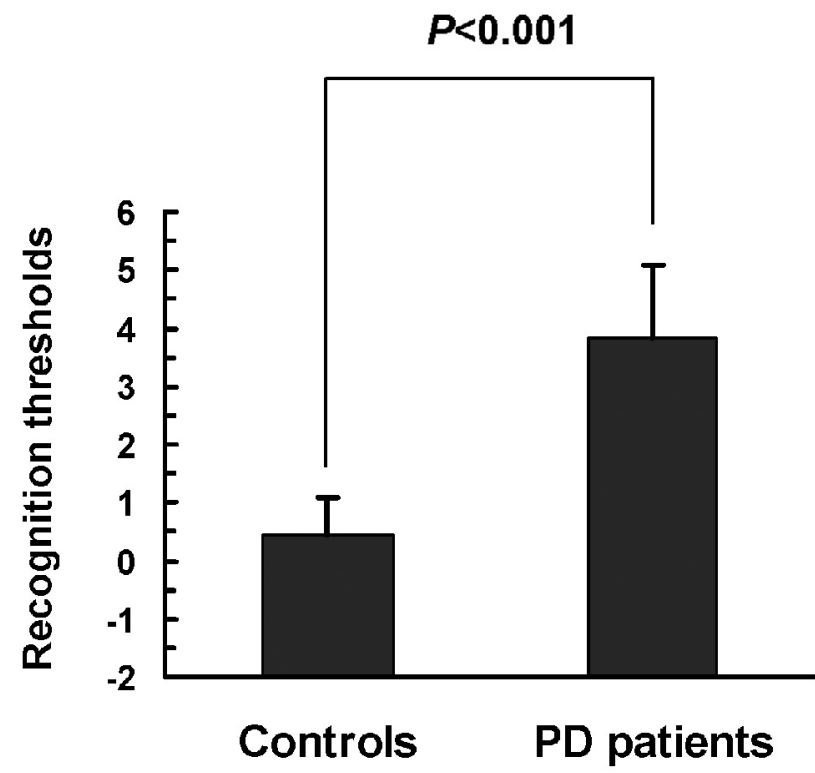

Fig 2. T\&T olfactometer threshold tests in patients with PD and in healthy controls. The means of recognition thresholds are significantly higher in patients with PD than in control subjects. Data are expressed as means \pm SEM.

\section{Statistical Analyses}

Data were expressed as mean \pm SEM. The various measures were assessed by using the Statistical Package for the Social Sciences, Version 11.5 (SPSS, Chicago, Illinois). Differences between patients with $\mathrm{PD}$ and healthy controls were analyzed by a paired-samples $t$ test. Relationships among olfactory function, the volume of $\mathrm{OB}$, and the depth of OS were examined by using Pearson correlation coefficients. Values of $P<.05$ were assumed to be statistically significant.

\section{Results}

\section{Olfactory Function Decreased in Patients with PD Compared with Healthy Controls}

Several tests have been used for olfactory assessment, including tests of odor identification, detection, discrimination, memory, suprathreshold odor intensity, and pleasantness perception. Previous studies have demonstrated that the test-retest reliability was equivalent for the T\&T olfactometer and the University of Pennsylvania Smell Identification Test. ${ }^{25,26}$ In this study, we used the T\&T olfactometer to evaluate the olfactory performance. As shown in Fig 2, the means of recognition thresholds were significantly higher in patients with $\mathrm{PD}$ than in control subjects $(3.82 \pm 1.25$ and $0.45 \pm 0.65$ respectively; $t=14.59, P<.0001)$.

\section{OB Volume and OS Depth Lower in Patients with PD Compared with Controls}

Numerous studies have demonstrated that OB volume assessed by MR imaging is correlated to olfactory function in healthy subjects and in patients with pathologic conditions. ${ }^{27}$ MR imaging was used to compare the structures of the $\mathrm{OB}$ and the OS in patients with PD and healthy controls. As shown in Fig 1, the OB and OS could be seen by using both T1-weighted and T2-weighted imaging sequences. Quantitative analyses showed that the volume of the OB varied from 26 to $90 \mathrm{~mm}^{3}$, with a mean of $44.87 \pm 11.84 \mathrm{~mm}^{3}$, in the control group (Fig 3 ). In contrast, the patients with PD had mean $\mathrm{OB}$ volume of
$37.30 \pm 10.23 \mathrm{~mm}^{3}$, which was statistically different from that of the controls $(t=2.98, P<.01)$. The mean depth of the OS was statistically smaller in patients with $\mathrm{PD}$ than in control subjects $(8.90 \pm 1.42 \mathrm{~mm}$ and $9.67 \pm 1.24 \mathrm{~mm}$, respectively; $t=2.32, P<.05)$.

\section{Correlations among Olfactory Function, $O B$ Volume, and OS Depth}

As shown in Fig 4, the volume of the OB correlated positively with odor recognition threshold scores as obtained by T\&T olfactometry in both patients with $\mathrm{PD}$ and in controls, $(r=$ -0.448 and $P<.0001$ for patients with PD; $r=-0.420, P<$ .05 for controls). In contrast, there was not a significant correlation between the depth of the OS and olfactory performance in either cohort $(r=-0.045, P=0.81$ for patients with $\mathrm{PD} ; r=-0.09, P=.61$ for controls).

\section{Discussion}

Although most patients with PD have olfactory deficits, ${ }^{1,2,13,18,19,28}$ the pathologic mechanism is unknown. The present study indicated that there was atrophy of the olfactory system in the patients with $\mathrm{PD}$, as shown by lower $\mathrm{OB}$ volume and OS depth compared with healthy controls. Most important, the OB volume correlated positively with olfactory dysfunction in patients with PD. This will provide insight into the critical interplay of olfactory functional loss and structural abnormalities in PD.

Idiopathic PD is traditionally considered a movement disorder, with hallmark lesions located in the SNpc. However, recent histopathologic studies suggest the possibility that $\mathrm{PD}$ is a multisystem disorder that progresses in a predictable sequence as described in the staging criteria of Braak et al. ${ }^{12,29}$ The disease process starts in the dorsal motor nucleus of the vagus and anterior olfactory nucleus and bulb and spreads through the brain stem nuclei to ultimately reach the SNpc, progressing through stages 1, 2, and 3. ${ }^{30}$ Early diagnosis of PD in stages 1 and 2, when degenerative changes have not yet reached the SNpc, may allow progression of the disease to be halted or delayed. Braak et $\mathrm{al}^{29,31}$ and Hawkes et $\mathrm{al}^{32}$ found that the initial changes in stage 1 of the disease start simultaneously in the dorsal nucleus of the vagus nerve and in the olfactory nucleus and bulb where the olfactory neuroepithelium is in the entry zone. It is possible that initial changes in the olfactory nucleus and bulb may contribute to the early loss of olfactory function observed in patients with PD.

The early diagnostic procedures incorporating olfactory evaluation such as the University of Pennsylvania Smell Identification Test $^{33}$ are already used. To further diagnose the nature of the olfactory deficit, MR imaging can be used. ${ }^{13,15-18} \mathrm{~A}$ recent DWI study confirmed disruption of the olfactory tract in patients with early stages of $\mathrm{PD},{ }^{34}$ and DWI would add an observer-independent and quantitative measure of symptoms in at-risk subjects. More recently, a morphometric analysis of MR imaging, called voxel-based morphometry, was used to investigate gray matter atrophy related to psychophysically measured scores of olfactory function in patients with early $\mathrm{PD}$, patients with moderately advanced PD, and age-matched healthy controls. ${ }^{13}$ The results provided first evidence that olfactory dysfunction in PD is related to atrophy in olfactoryeloquent regions of the limbic and paralimbic cortex. 

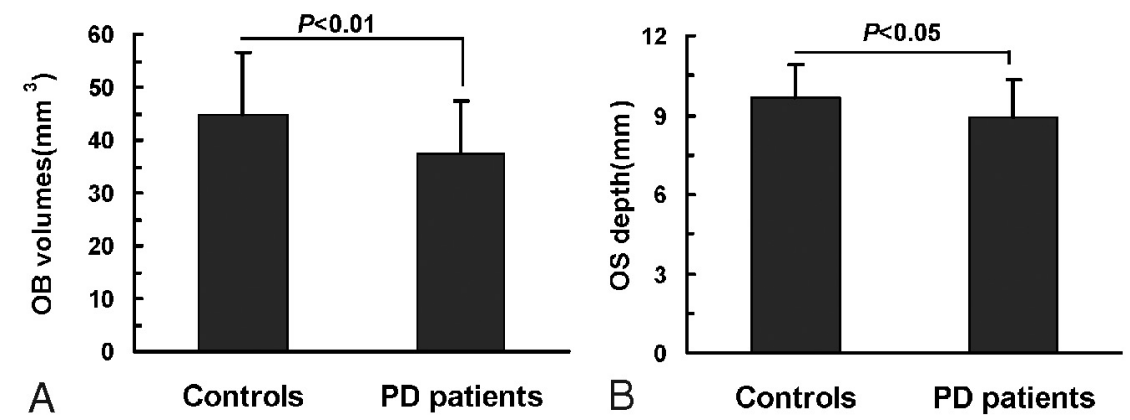

Fig 3. $\mathrm{OB}$ volume $(A)$ and $\mathrm{OS}$ depth $(B)$ in patients with $\mathrm{PD}$ and in healthy controls. The mean $\mathrm{OB}$ volumes and the mean $\mathrm{OS}$ depths are statistically smaller in patients with PD than in control subjects. Data are expressed as means \pm SEM.
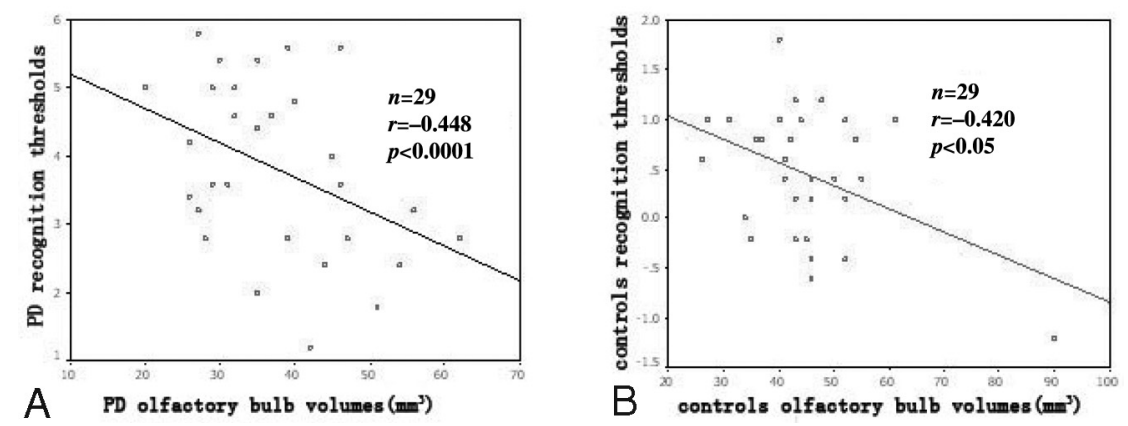

Fig 4. Correlation of the volume of $\mathrm{OB}$ with olfactory performance in patients with $\mathrm{PD}(A)$ and in healthy controls $(B)$. In patients with $\mathrm{PD}$ and in controls, the $\mathrm{OB}$ volumes correlate positively with odor recognition threshold scores.

Recently, several studies have been set out to investigate the structural changes at the level of the $\mathrm{OB}$ and $\mathrm{OS}$ in patients with $\mathrm{PD} .{ }^{15-18}$ The $\mathrm{OB}$ ends with the olfactory tract and is closely related to the OS of the frontal lobe. The OB collects the sensory afferents of the olfactory receptor cells located in the olfactory neuroepithelium. It has been shown that $\mathrm{OB}$ volume decreases with the duration of the olfactory loss. ${ }^{27}$ However, comparison of $\mathrm{OB}$ volumes did not yield significant differences between patients with PD $(n=11)$ with anosmia and healthy normosmic controls. ${ }^{15}$ Support for the results of a nonsignificant difference in $\mathrm{OB}$ volume between patients with PD and controls comes from reports by Mueller et $\mathrm{al}^{18}$ and Huisman et al, ${ }^{9}$ though there were only 11 and 10 patients with PD in these studies, respectively. In the present study, 29 patients with PD and 29 age- and sex-matched healthy controls were included, providing more statistical power. Because olfaction may be processed predominantly in the right hemisphere, ${ }^{23,35,36}$ both sides of each individual were measured, and the greater $\mathrm{OB}$ volume was used in our analysis, in which we observed significant differences in $\mathrm{OB}$ volume between patients with PD and controls. In addition, the OS depth was significantly smaller in patients with PD than in controls; this result differed from that of another study in which no difference in OS depth was found between patients with PD ( $n=$ 42) and controls. ${ }^{16}$ Therefore, further studies of more subjects are needed.

Our study using the T\&T olfactometer threshold test confirmed that patients with PD experience olfactory loss, consistent with previous studies. ${ }^{2,19}$ The correlation of olfactory function with volume of the human $\mathrm{OB}$ has been established. ${ }^{24,37}$ In this study, a positive correlation between olfactory deficit and $\mathrm{OB}$ volume was found both in patients with
PD and controls. It may be that olfactory dysfunction in PD is related to loss of olfactory neurons or disrupted input to the $\mathrm{OB}$. We found no correlation of olfactory performance and OS depth, indicating that olfactory loss does not parallel the structural changes to the OS in patients with PD. Together, these data support the idea that olfactory loss in the earliest stages of PD (stage 1 according to Braak et $\mathrm{al}^{12}$ ) is not a consequence of damage to the $\mathrm{OS}$ but rather results from $\mathrm{OB}$ atrophy.

\section{Conclusions}

In summary, the present study was performed with a large sample size, well-validated test instruments, standardized neurologic examinations, and published diagnostic criteria. The results provide evidence that olfactory impairment is a valuable biomarker for PD. Olfactory testing together with neuroimaging of olfactory structures may provide a simple and relatively economic means of identifying individuals at high risk for development of PD.

\section{Acknowledgments}

We thank Medjaden Bioscience Limited for assisting in the preparation of this article.

\section{References}

1. Ansari KA, Johnson A. Olfactory function in patients with Parkinson's disease. J Chronic Dis 1975;28:493-97

2. Doty RL, Deems DA, Stellar S. Olfactory dysfunction in parkinsonism: a general deficit unrelated to neurologic signs, disease stage, or disease duration. Neurology 1988;38:1237-44

3. Mesholam RI, Moberg PJ, Mahr RN, et al. Olfaction in neurodegenerative disease: a meta-analysis of olfactory functioning in Alzheimer's and Parkinson's diseases. Arch Neurol 1998;55:84-90 
4. Daum RF, Sekinger B, Kobal G, et al. Olfactory testing with "sniffin' sticks" for clinical diagnosis of Parkinson disease [in German]. Nervenarzt 2000;71:643-50

5. Herting B, Schulze S, Reichmann $\mathrm{H}$, et al. A longitudinal study of olfactory function in patients with idiopathic Parkinson's disease. J Neurol 2008;255:367-70

6. Hawkes $\mathrm{CH}$, Shephard $\mathrm{BC}$, Daniel SE. Is Parkinson's disease a primary olfactory disorder? QJM 1999;92:473-80

7. Del Tredici K, Rub U, De Vos RA, et al. Where does Parkinson disease pathology begin in the brain? J Neuropathol Exp Neurol 2002;61:413-26

8. Ross GW, Abbott RD, Petrovitch H, et al. Association of olfactory dysfunction with incidental Lewy bodies. Mov Disord 2006;21:2062-67

9. Huisman E, Uylings HB, Hoogland PV. A $\mathbf{1 0 0 \%}$ increase of dopaminergic cells in the olfactory bulb may explain hyposmia in Parkinson's disease. Mov Disord 2004;19:687-92

10. Pearce RK, Hawkes CH, Daniel SE. The anterior olfactory nucleus in Parkinson's disease. Mov Disord 1995;10:283-87

11. Silveira-Moriyama L, Holton JL, Kingsbury A, et al. Regional differences in the severity of Lewy body pathology across the olfactory cortex. Neurosci Lett 2009; $453: 77-80$

12. Braak H, Del Tredici K, Rub U, et al. Staging of brain pathology related to sporadic Parkinson's disease. Neurobiol Aging 2003;24:197-211

13. Wattendorf E, Welge-Lussen A, Fiedler K, et al. Olfactory impairment predicts brain atrophy in Parkinson's disease. J Neurosci 2009;29:15410-13

14. Hoogland PV, van den Berg R, Huisman E. Misrouted olfactory fibres and ectopic olfactory glomeruli in normal humans and in Parkinson and Alzheimer patients. Neuropathol Appl Neurobiol 2003;29:303-11

15. Hummel T, Witt M, Reichmann H, et al. Immunohistochemical, volumetric, and functional neuroimaging studies in patients with idiopathic Parkinson's disease. J Neurol Sci 2010;289:119-22

16. Kim JY, Lee WY, Chung EJ, et al. Analysis of olfactory function and the depth of olfactory sulcus in patients with Parkinson's disease. Mov Disord 2007;22:1563-66

17. Held P, Seitz J, Frund R, et al. MRI detection of olfactory bulb and tract. J Neuroradiol 2000;27:112-18

18. Mueller A, Abolmaali ND, Hakimi AR, et al. Olfactory bulb volumes in patients with idiopathic Parkinson's disease: a pilot study. J Neural Transm 2005;112:1363-70

19. Hawkes CH, Shephard BC, Daniel SE. Olfactory dysfunction in Parkinson's disease. J Neurol Neurosurg Psychiatry 1997;62:436-46

20. Gelb DJ, Oliver E, Gilman S. Diagnostic criteria for Parkinson disease. Arch Neurol 1999;56:33-39
21. Zusho H, Asaka H, Okamoto M. Diagnosis of olfactory disturbance. Auris Nasus Larynx 1981;8:19-26

22. Hummel T, Damm M, Vent J, et al. Depth of olfactory sulcus and olfactory function. Brain Res 2003;975:85-89

23. Abolmaali ND, Hietschold V, Vogl TJ, et al. MR evaluation in patients with isolated anosmia since birth or early childhood. AJNR Am J Neuroradiol 2002;23:157-64

24. Haehner A, Rodewald A, Gerber JC, et al. Correlation of olfactory function with changes in the volume of the human olfactory bulb. Arch Otolaryngol Head Neck Surg 2008;134:621-24

25. Doty RL, McKeown DA, Lee WW, et al. A study of the test-retest reliability of ten olfactory tests. Chem Senses 1995;20:645-56

26. Kondo H, Matsuda T, Hashiba M, et al. A study of the relationship between the T\&T olfactometer and the University of Pennsylvania Smell Identification Test in a Japanese population. Am J Rhinol 1998;12:353-58

27. Rombaux P, Duprez T, Hummel T. Olfactory bulb volume in the clinical assessment of olfactory dysfunction. Rhinology 2009;47:3-9

28. Haehner A, Hummel T, Reichmann H. Olfactory dysfunction as a diagnostic marker for Parkinson's disease. Expert Rev Neurother 2009;9:1773-79

29. Braak H, Rub U, Gai WP, et al. Idiopathic Parkinson's disease: possible routes by which vulnerable neuronal types may be subject to neuroinvasion by an unknown pathogen. J Neural Transm 2003;110:517-36

30. Lerner A, Bagic A. Olfactory pathogenesis of idiopathic Parkinson disease revisited. Mov Disord 2008;23:1076-84

31. Braak H, Ghebremedhin E, Rub U, et al. Stages in the development of Parkinson's disease-related pathology. Cell Tissue Res 2004;318:121-34

32. Hawkes $\mathrm{CH}$, Del Tredici K, Braak H. Parkinson's disease: a dual-hit hypothesis. Neuropathol Appl Neurobiol 2007;33:599-614

33. Doty RL, Shaman P, Dann M. Development of the University of Pennsylvania Smell Identification Test: a standardized microencapsulated test of olfactory function. Physiol Behav 1984;32:489-502

34. Scherfler C, Schocke MF, Seppi K, et al. Voxel-wise analysis of diffusion weighted imaging reveals disruption of the olfactory tract in Parkinson's disease. Brain 2006;129:538-42

35. Zatorre RJ, Jones-Gotman M, Evans AC, et al. Functional localization and lateralization of human olfactory cortex. Nature 1992;360:339-40

36. Sobel N, Khan RM, Saltman A, et al. The world smells different to each nostril. Nature 1999;402:35

37. Buschhuter D, Smitka M, Puschmann S, et al. Correlation between olfactory bulb volume and olfactory function. Neuroimage 2008;42:498-502 